CARDIOVASCULAR MEDICINE

\title{
Transoesophageal echocardiographic assessment of mitral valve commissural morphology predicts outcome after balloon mitral valvotomy
}

\author{
N Sutaria, T R D Shaw, B Prendergast, D Northridge
}

Heart 2006;92:52-57. doi: 10.1136/hrt.2004.058297

See end of article for authors' affiliations

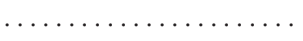

Correspondence to: Dr Nilesh Sutaria, Royal Brompton Hospital, Sydney Street, London sW3 6NP, UK; NSutaria@ doctors.org.uk

Accepted 4 March 2005
Objective: To investigate the value of transoesophageal echocardiography in the assessment of commissural morphology and prediction of outcome after balloon mitral valvotomy (BMV).

Design: Prospective study.

Setting: Tertiary cardiac referral centre.

Patients: 72 consecutive patients (mean age 61.3 years, range 38-89 years) referred for BMV.

Interventions: Transoesophageal echocardiography was performed immediately before BMV and the mitral commissures were scanned systematically. Anterolateral and posteromedial commissures were scored individually according to whether non-calcified fusion was absent (0), partial (1), or extensive (2). Calcified commissures usually resist splitting and scored 0 . Scores for each commissure were combined giving an overall commissure score for each valve of $0-4$, higher scores reflecting increased likelihood of commissural splitting. Valve anatomy was also graded by the method of Wilkins et al, which does not include commissural assessment.

Main outcome measures: Patients were divided into outcome groups: A (good) and B (suboptimal). "Good" was defined as final valve area $>1.5 \mathrm{~cm}^{2}$ with a $>25 \%$ increase in area and absence of severe mitral regurgitation judged by echocardiography.

Results: Valve area increased from a mean (SD) of $1.1(0.28) \mathrm{cm}^{2}$ to $1.8(0.46) \mathrm{cm}^{2}$. Commissure scores were higher in group $A$ than in group $B(p<0.01)$, scores $\geqslant 2$ predicting a good outcome with positive and negative accuracy of $67 \%$ and $82 \%$, respectively $(p<0.001)$. Commissure score was the strongest independent predictor of outcome.

Conclusion: Transoesophageal echocardiographic assessment of commissural morphology predicts outcome after BMV, adding significantly to the Wilkins score.
S ince its introduction in 1984 by Inoue et al, ${ }^{1}$ balloon mitral valvotomy (BMV) has become established as a safe and effective treatment for rheumatic mitral stenosis with equivalent results to surgical valvotomy. ${ }^{2-4}$ In vivo and in vitro studies have confirmed that commissural splitting is the dominant mechanism by which mitral valve area (MVA) is increased during balloon dilatation..$^{5-7}$ In the selection of patients for BMV, mitral valve morphology is conventionally assessed by two dimensional transthoracic echocardiography (TTE), leading to an echocardiographic score based on the criteria of Wilkins et al. ${ }^{8}$ The Wilkins score is based on a semiquantitative assessment of leaflet thickening, mobility, calcification, and subvalvar thickening. Each is graded on a scale of 0 or 1 to 4 . Higher Wilkins scores $(>8)$ indicate more severe degenerative disease and studies have shown this to be a significant though relatively weak predictor of success from BMV. ${ }^{8}$ However, the Wilkins score does not include commissural assessment. Recent studies have shown the assessment of commissural morphology by TTE to be of value in predicting outcome after BMV. In 149 patients investigated by Cannan et al, ${ }^{10}$ the mitral commissures were assessed in the short axis parasternal view. Commissural calcification was associated with a lower survival rate and a higher incidence of mitral valve replacement after BMV. Fatkin et al studied 30 patients by a similar transthoracic approach and showed that an assessment of commissural disease before BMV is useful for the prediction of commissural splitting and the final increase in valve area. We previously found in a transthoracic study of 300 patients that commissural calcification was an independent predictor of outcome with only $40 \%$ of those with commissural calcification achieving a final MVA $>1.5 \mathrm{~cm}^{2}$ compared with $64 \%$ of those without commissural calcification. ${ }^{11}$ However, elderly and obese patients were sometimes poor subjects for TTE.

Transoesophageal echocardiography (TOE) is now performed routinely in most centres before BMV, primarily to exclude left atrial thrombus. In comparison with TTE, TOE offers superior resolution of the mitral valve apparatus and overcomes problems caused by anterior leaflet shadowing, thereby allowing more accurate localisation of mitral calcification. We have developed a novel TOE method that provides a systematic scan of the whole length of the mitral commissure. The objectives of this prospective study were, firstly, to evaluate this new TOE technique in assessing mitral commissural fusion and calcification; secondly, to devise a commissure score based on TOE assessment to reflect the likelihood of commissural splitting; thirdly, to determine the accuracy of this commissure score in predicting immediate outcome after BMV; fourthly, to investigate the accuracy of TTE assessment of mitral commissural morphology compared with TOE in a subset of patients; and lastly, to compare the commissure score derived by TOE with the established Wilkins score.

Abbreviations: $B M V$, balloon mitral valvotomy; $M R$, mitral regurgitation; MVA, mitral valve area; $\mathrm{TOE}$, transoesophageal echocardiography; TTE, transthoracic echocardiography 


\section{METHODS}

\section{Patients}

The series consisted of 72 patients with symptomatic mitral stenosis undergoing BMV at the Western General Hospital, Edinburgh. This was an elderly population, with a mean age 61.3 years (range $38-89$ years). The majority $(60,83 \%)$ were women and $51(71 \%)$ were in atrial fibrillation. Fifteen patients $(21 \%)$ had presented with restenosis after surgical valvotomy. In eight patients judged inoperable by the referring physician or cardiac surgeon, BMV was performed as a palliative treatment.

\section{Echocardiographic study}

Transthoracic echocardiography

All patients underwent two dimensional and Doppler TTE immediately before BMV with Hewlett Packard Sonos 2000, Acuson $128 \mathrm{XP} / 10$, or ATL Ultramark 9 ultrasound scanners. Diastolic transmitral flow was analysed by pulsed Doppler with the sample volume at the tips of the mitral leaflets in the apical four chamber view. Initial MVA was measured by Doppler pressure half time taking the mean of five recordings. The severity of mitral regurgitation (MR) after BMV was assessed by colour and continuous wave Doppler.

Final MVA was measured by either planimetry of the mitral orifice in the parasternal short axis view within 24 hours after BMV or by Doppler pressure half time at one month's follow up. A good outcome was defined as final MVA $>1.5 \mathrm{~cm}^{2}$ and an increase in valve area $>25 \%$ of the baseline value in the absence of severe MR on echocardiography.

Commissural morphology was assessed by TTE in the parasternal short axis view as described previously. ${ }^{71}$ Both anterolateral and posteromedial commissures were individually examined. Calcium was identified by bright, confluent echoes. Commissural calcification was judged to be present if echocardiographic density was brighter within the commissures than in the adjacent aortic root (fig 1).

\section{Transoesophageal echocardiography}

All patients underwent TOE immediately before BMV with a $7 \mathrm{MHz}$ multiplane transoesophageal probe and Acuson 128 $\mathrm{XP} / 10$ ultrasound scanner. The examination was performed after a four hour fast under sedation with midazolam. The mitral valve leaflets and subvalvar apparatus were examined at mid-oesophageal and transgastric levels. Mitral valve anatomy was scored according to the criteria of Wilkins et al. ${ }^{8}$ The mitral commissures were scanned systematically at the mid-oesophageal level. The anterolateral commissure was visualised in the transverse plane by advancing and retracting

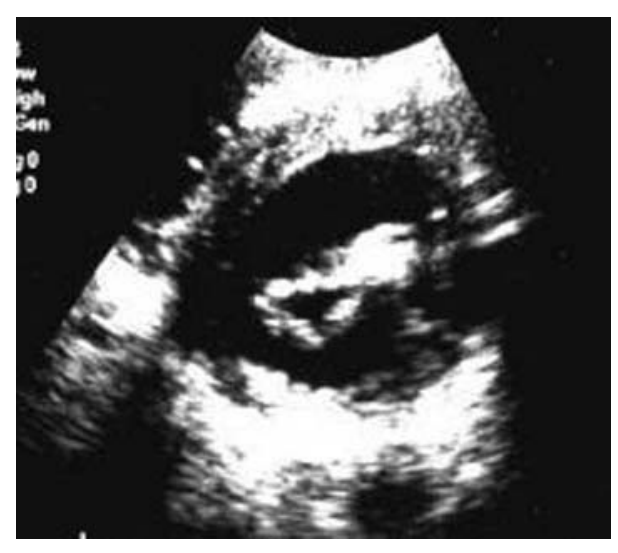

Figure 1 Calcified anterolateral commissure seen in the transthoracic short axis parasternal view. the probe, thereby scanning the length of the commissure from the leaflet tips to the annulus. The posteromedial commissure was scanned in the longitudinal plane by rotating the probe clockwise and anticlockwise (fig 2). Commissural calcification was identified by high intensity echoes casting an acoustic shadow (fig 3). Each commissure was assigned a score to reflect the likelihood of splitting. A score of 0 was given if there was no fusion or if commissural calcification, expected to resist splitting, was present. When non-calcified fusion was present, a score of 1 was given if the fusion was estimated to extend no more than $5 \mathrm{~mm}$ from the annulus. A score of 2 was given when fusion extended $>5 \mathrm{~mm}$ from the annulus towards the centre of the valve. Scores for anterolateral and posteromedial commissures were combined such that each valve had an overall commissure score ranging from 0-4. A high score indicated extensively fused, non-calcified commissures that were therefore more likely to split. A low score indicated either minimal fusion or the presence of resistant commissural calcification. Table 1 summarises the system for deriving the commissure score.

\section{Interobserver variability of TOE assessment}

Two independent observers blinded to the outcome of BMV retrospectively reviewed TOE images of 30 patients $(60$ commissures). Agreement between observers was good in the scoring of each commissure $(\kappa=0.73$ for anterolateral and $\kappa=0.75$ for posteromedial commissures). Observers agreed on the presence or absence of commissural calcification in all but one commissure ( $98 \%$ concordance, $\kappa=0.95$ )

\section{Comparison between TOE and TTE assessment of commissural calcification}

In a subset of 33 patients, transthoracic assessment of commissural morphology was compared with TOE assessment. Video recordings were retrospectively analysed by consensus of two observers. In these elderly patients with degenerative valve disease, optimal visualisation of the commissures was not always possible and six of the 33 patients $(18 \%)$ were excluded because of inadequate transthoracic images. The majority of these patients had been scanned on earlier ultrasound machines without harmonic imaging capability. The extent of commissural fusion is difficult to quantify reliably by the transthoracic technique and therefore only commissural calcification was evaluated. With TOE used as the reference standard, in the remaining 27 patients TTE correctly detected 11 of 12 calcified commissures (sensitivity of 92\%). TTE incorrectly localised leaflet calcification to the commissure in seven patients (specificity 83\%). Interobserver variation for identification of commissural calcification by TTE was $7 \%$.

\section{Technique of BMV}

BMV was performed percutaneously under local anaesthetic by the same primary operator (TRDS) using the Inoue technique. Balloon size was selected according to the patient's height. The balloon was inflated stepwise under fluoroscopic guidance until the reduction in transmitral gradient was satisfactory. After each inflation the degree of MR was assessed by left ventriculography and the balloon was not inflated further if significant MR had developed.

\section{Statistical analysis}

Data are expressed as mean (SD). Haemodynamic data before and after BMV were compared by the paired Student's $t$ test. Non-parametric data and binary factors were assessed with respect to outcome by the Mann-Whitney and $\chi^{2}$ tests, respectively. Stepwise multiple linear regression analysis was used to determine the importance of age, commissural calcification, sex, rhythm, commissure score, and Wilkins 


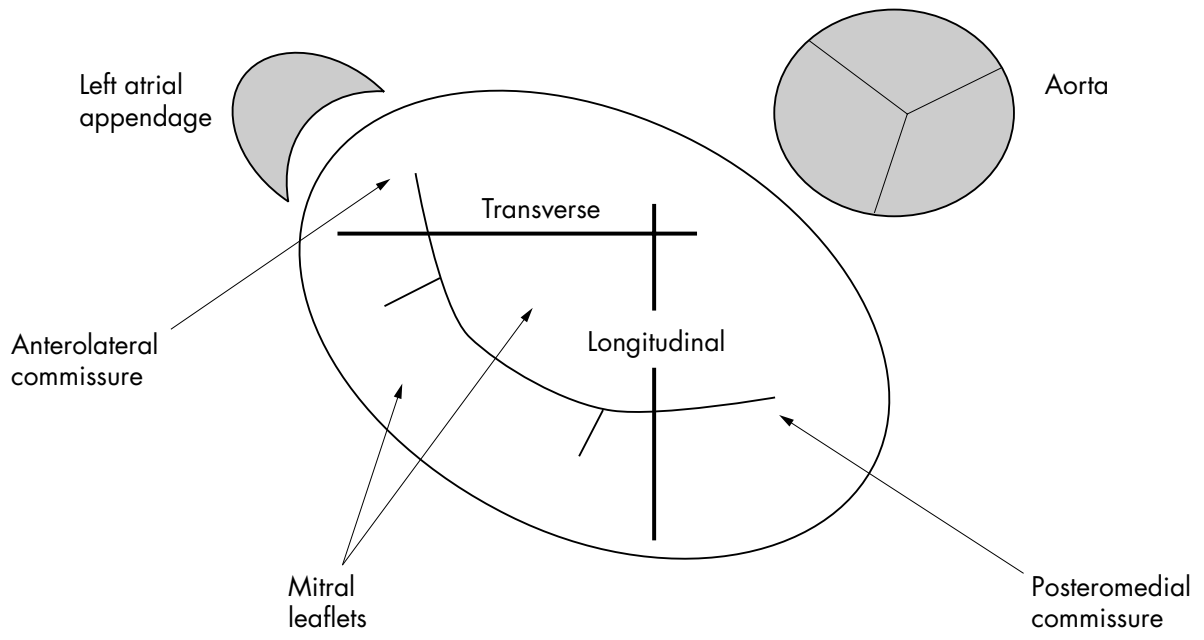

Figure 2 Mitral valve anatomy viewed from the left atrium with orientation of the transverse and longitudinal transoesophageal planes.

score as independent predictors of outcome. The correlation between commissure score and gain in MVA was studied by the Kendall rank analysis. A probability value of $\mathrm{p}<0.05$ was considered significant. A commercially available statistics software package (SPSS for Microsoft Windows; SPSS Inc, Chicago, Illinois, USA) was used for all calculations.

\section{RESULTS}

\section{Outcome from BMV}

The mean Wilkins score was 6.6 (range 3-12); 17 patients (24\%) had a Wilkins score $>8$ predicting an unfavourable outcome from BMV. Balloon dilatation of the mitral valve was achieved in all patients and mean MVA assessed by echocardiography increased from $1.1(0.28) \mathrm{cm}^{2}$ to 1.8 $(0.46) \mathrm{cm}^{2}(\mathrm{p}<0.01)$. Invasive measurements based on the Gorlin formula showed MVA to increase from 0.98 $(0.29) \mathrm{cm}^{2}$ to $1.74(0.54) \mathrm{cm}^{2}$.

Eight patients (11\%) developed severe MR on echocardiography. Forty patients (56\%) achieved a good outcome, as defined by our prespecified criteria. Patients were divided according to outcome into groups A (good) and B (suboptimal).

\section{Commissure score}

The commissure score and outcome after BMV were significantly correlated, higher scores predicting a more

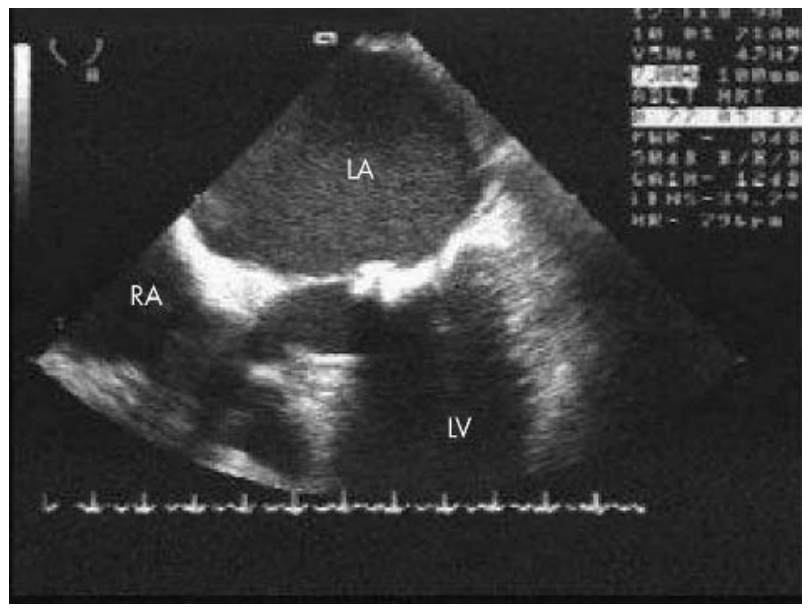

Figure 3 Calcified anterolateral commissure seen in the transverse midoesophageal plane. LA, left atrium; LV, left ventricle; RA, right atrium. favourable result (table 2, fig 4). Thirty seven of 55 patients with a commissure score of $\geqslant 2$ obtained a good outcome compared with only three patients of 17 with a commissure score of 0 or 1 (positive and negative predictive accuracy $67 \%$ and $82 \%$, respectively, $\mathrm{p}<0.01$ ).

\section{Commissural calcification}

Twenty four patients $(33.3 \%)$ had calcification localised to either the anterolateral or posteromedial commissure by TOE. No patients had bicommissural calcification. Commissural calcification predicted a suboptimal result, being present in $18 \%$ of group A patients compared with $53 \%$ of group B $(\mathrm{p}<0.01)$. Commissural calcification increased significantly with advancing age $(\mathrm{p}<0.001)$. Patients with higher

Table 1 Commissure score based on transoesophageal echocardiographic assessment

\begin{tabular}{|c|c|}
\hline $\begin{array}{l}\text { Commissure } \\
\text { score }\end{array}$ & Commissure morphology \\
\hline 0 & $\begin{array}{l}\text { Neither commissure fused } \\
\text { or calcification of both commissures } \\
\text { or absent fusion of one commissure and calcification of } \\
\text { the other }\end{array}$ \\
\hline 1 & $\begin{array}{l}\text { Partial fusion of one commissure and absent fusion or } \\
\text { calcification of the other }\end{array}$ \\
\hline 2 & $\begin{array}{l}\text { Extensive fusion of one commissure and absent fusion or } \\
\text { calcification of the other } \\
\text { or partial fusion of both commissures }\end{array}$ \\
\hline 3 & $\begin{array}{l}\text { Extensive fusion of one commissure, partial fusion of the } \\
\text { other, and no commissural calcification }\end{array}$ \\
\hline 4 & $\begin{array}{l}\text { Extensive fusion of both commissures and no commissural } \\
\text { calcification }\end{array}$ \\
\hline
\end{tabular}

Table 2 Relation between commissure score and outcome

\begin{tabular}{llll}
\hline & & \multicolumn{2}{l}{ Outcome group } \\
\cline { 3 - 4 } Commissure score & No & A (good) & B (suboptimal) \\
\hline 0 & 1 & 0 & 1 \\
1 & 16 & $3(19 \%)$ & 13 \\
2 & 18 & $10(56 \%)$ & 8 \\
3 & 20 & $14(70 \%)$ & 6 \\
4 & 17 & $13(76 \%)$ & 4 \\
\hline$p<0.01\left(\chi^{2}\right)$. & & &
\end{tabular}




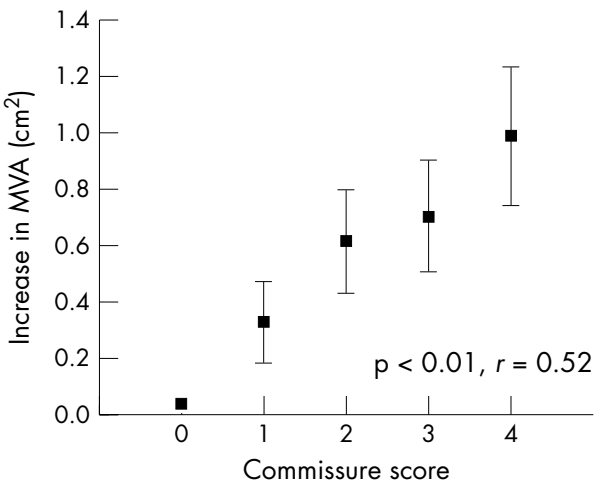

Figure 4 Correlation between commissure score and increase in mitral valve area (MVA) after balloon mitral valvotomy.

Wilkins scores were more likely to have commissural calcification $(p=0.013)$. Commissural calcification and patients' sex or baseline valve area were not significantly correlated.

\section{Wilkins score}

The Wilkins score significantly predicted outcome (mean Wilkins score 5.9 (2.1) $v 7.4$ (2.0) in groups A and B, respectively, $\mathrm{p}<0.01$ ). Patients achieving a greater increase in valve area tended to have lower Wilkins scores. At the conventional cut off of 8, the Wilkins score was less helpful in predicting good outcome compared with the commissure score (positive and negative accuracy of $60 \%$ and 59\%, respectively, not significant). The Wilkins score and commissure score were not significantly correlated.

\section{Multivariate analysis}

In a multivariate linear regression model, which included age, sex, rhythm, commissure score, commissural calcification, and Wilkins score, both the commissure score (odds ratio $1.19, \mathrm{p}=0.001$ ) and the Wilkins score (odds ratio 0.94, $\mathrm{p}=0.012$ ) were shown to be independent predictors of good outcome from BMV.

\section{Combined assessment of Wilkins criteria and commissural morphology}

Combining the evaluation of splittable, non-calcified commissural fusion and thickening, restriction, and calcification in the leaflets and subvalvar apparatus helped to refine the prediction of outcome further. In patients with good Wilkins valves (Wilkins score $\leqslant 8$ ), leaflet and subvalvar changes are minimal and severe stenosis is due predominantly to commissural fusion. In such cases the commissure score (assessing non-calcified, splittable commissural fusion) was more accurate in predicting outcome. In this group of patients a commissure score $\geqslant 2$ predicted a good result with positive and negative predictive value of $75 \%$ and $80 \%$, respectively. In those with bad Wilkins valves (Wilkins score $>8$ ) only $47 \%$ of those with a commissure score $\geqslant 2$ obtained a good result.

\section{Mitral regurgitation}

We did not observe a significant correlation between commissure score and the development of severe echocardiographic MR. The eight patients developing severe MR had a significantly higher Wilkins score (8.4 (1.9) v 6.3 (2.1), $\mathrm{p}<0.02)$. We observed that four of these eight patients had commissural calcification: in these four cases TTE in the short axis parasternal plane showed the mechanism of MR to be a leaflet tear adjacent to the area of calcification.

\section{DISCUSSION}

Mitral valve anatomy has been shown to be the strongest independent predictor of success after BMV. ${ }^{49}$ The Wilkins echocardiographic score ${ }^{8}$ is used widely to guide patient selection for BMV. This score is based on an assessment of leaflet thickness, mobility, and calcification and of the extent of subvalvar disease, but commissural morphology is not directly examined. A significant subset of patients with high Wilkins scores still attain a good result after balloon valvotomy. ${ }^{12}{ }^{13}$ In an early study evaluating the relation between Wilkins score and change in MVA, Abascal et al ${ }^{9}$ observed a substantial scatter in the data; although $84 \%$ of those with scores $\leqslant 8$ had a good outcome, so did $42 \%$ of those with scores $>8$. More recent studies have found this scoring system to be an imprecise predictor of outcome, ${ }^{7}{ }^{10}$ indicating the need for more refined echocardiographic assessment.

In vivo and in vitro studies ${ }^{5-7}$ have confirmed that the mechanism underlying the increase in valve area associated with BMV involves splitting of one or both fused mitral commissures in a manner similar to surgical commissurotomy. BMV is therefore unlikely to increase the MVA if commissural fusion is absent or minimal, or if the commissures resist splitting because of the presence of calcium. In the present study, commissural calcification was identified in a third of our elderly population and predicted a suboptimal immediate outcome. The significance of commissural calcification has recently been reported by other investigators. In 149 patients investigated by Cannan et al ${ }^{10}$ the mitral commissures were examined for the presence or absence of calcification by TTE alone in the parasternal short axis view. Calcification localised to a commissure was associated with a lower mid term survival rate and a higher incidence of mitral valve replacement. Fatkin et $a l^{7}$ used a similar transthoracic approach in 30 patients undergoing BMV. Commissural splitting resulted in a greater increase in valve area and occurred if at least one commissure was not heavily fibrosed or calcified. The assessment of commissural morphology allowed an accurate prediction of immediate outcome. Our own institution has reported the relation between commissural calcification and immediate outcome after BMV in 300 patients, mean age 61 years, studied by TTE. ${ }^{11}$ Only $40 \%$ of those with commissural calcification visualised in the parasternal short axis view achieved a final MVA $>1.5 \mathrm{~cm}^{2}$ compared with $64 \%$ of those without. A quantitative grading of the extent of commissural calcification was an independent predictor of outcome. This grading was most useful for patients with low Wilkins scores and therefore of little use for other degenerative disease of the leaflets and chordae.

Although the Wilkins score does not specifically assess the commissures, the Wilkins score and commissural calcification were significantly correlated. Degenerative disease of the commissures probably progresses in parallel with leaflet and subvalvar disease. Heavy generalised leaflet calcification is likely to involve the commissures. The Wilkins score therefore is a useful screening tool for patients referred for BMV and in our study was a significant independent predictor of outcome. However, our results and those of Fatkin et $a l^{7}$ and Cannan et al ${ }^{10}$ have shown that echocardiographic assessment of commissural morphology has important additional predictive value. In our patients, the commissure score was a more accurate predictor of outcome than the Wilkins score.

Two dimensional TTE remains the most commonly used technique for the assessment of mitral valve morphology and provides a screening tool for the selection of patients for BMV. In the present study, TTE was shown to have a high sensitivity (92\%) for detecting commissural calcium. However, in a significant proportion of patients (18\%), the commissures could not be adequately visualised. Leaflet 
calcium tended to be incorrectly localised to within the commissure, which reduced the specificity (83\%). Many centres routinely perform TOE before BMV. Transoesophageal imaging offers improved resolution and more accurate localisation of calcification than TTE. The extent of non-calcified commissural fusion was also semiquantitatively assessed by a previously unreported system. Calcified commissures were shown to be strongly associated with adverse outcome. In patients where one commissure was calcified, absent, or minimal, fusion of the opposite commissure (commissure score 0 or 1) resulted in a good outcome in only $18 \%$. In the absence of commissural calcium, a low commissure score of 0 or 1 may also be given when mitral stenosis is mild or when stenosis is caused by disease localised to the mitral leaflets or subvalvar apparatus rather than the commissures, a situation not infrequent after a previous surgical valvotomy. In both cases, balloon dilatation is unlikely to achieve a satisfactory increase in valve area. Evaluation of commissural fusion as well as calcification should therefore provide a scoring system that more accurately reflects valve splittability. We found a strong correlation between commissure score and increase in valve area ( $\mathrm{p}<0.001$; fig 4$)$, consistent with this hypothesis.

Our scoring system did not differentiate between nonfusion and calcification; in both instances a score of 0 would be assigned to the commissure, since we assume the commissure will not split at this site. Eighteen patients had a commissure score of 2 . Ten cases were caused by severe fusion of one commissure and calcification of the other: of these, four $(40 \%)$ had a good result. Five cases were caused by severe fusion of one commissure and absent fusion of the other: four $(80 \%)$ had a good result. Although patient numbers are small, the findings suggest that patients with one severely fused and splittable commissure and non-fusion at the other may still gain a favourable immediate result from balloon dilatation, whereas any commissural calcification confers an adverse prognosis.

TOE is a semi-invasive technique and therefore clear benefits must be seen to justify its routine use before BMV. Some studies have compared the relative merits of TTE and TOE in the assessment of patients undergoing BMV. Left atrial thrombus cannot be reliably excluded by TTE because of its inability to visualise the left atrial appendage fully in all patients, ${ }^{14-16}$ and many centres now routinely perform TOE before BMV for this reason. The longitudinal transgastric plane, when available, also allows more detailed visualisation of the chordae and papillary muscles than does TTE. ${ }^{17}$ TOE also has a role after BMV, since it is more accurate in determining the severity and mechanism of MR and in evaluating the degree of any residual left to right shunting across the interatrial septum. ${ }^{14} 17$

However, the specific value of TOE in the assessment of commissural morphology has not been reported previously. Other investigators advocate the use of the transgastric window to obtain images corresponding to the short axis parasternal view of the valve orifice. However, the mitral valve is conical so the commissures do not lie in a single plane and poor quality images are obtained when there is major left atrial dilatation. Consequently, this technique has not been shown to provide adequate visualisation of commissural calcification and fusion. ${ }^{16}{ }^{17}$ In the mid oesophagus, posterior to the left atrium, the mitral valve can be scanned at high frequency and is clearly visualised in all patients. We advocate systematic scanning of the anterolateral and posteromedial commissures in the transverse and longitudinal planes, respectively. A further advantage of TOE over TTE is that acoustic shadowing from leaflet calcification is cast behind the valve into the left ventricle and is less likely to obscure the orifice, thereby allowing more accurate localisation of calcification. The present study is the first to report the advantages of TOE over conventional TTE for the assessment of commissural calcification and fusion and to show the value of TOE in predicting procedural outcome after BMV.

It remains that $33 \%$ of patients with a high commissure score $(2,3$, or 4$)$ still failed to achieve a good outcome. This reflects the strict criteria for success used in our study and suggests that outcome is influenced by multiple aspects of mitral valve morphology. A combined assessment of favourable anatomical features (non-calcified commissural fusion) and unfavourable anatomical features (leaflet and subvalvar change, commissural calcification) is likely to be the best approach for evaluating patients referred for BMV. In accordance, the positive predictive value of the commissure score was improved in those patients with good Wilkins valves in whom commissural fusion is the dominant cause of stenosis.

Significant MR is a relatively common complication of BMV but one that remains difficult to predict from baseline anatomical and clinical variables. ${ }^{18}$ MR often increases mildly after BMV at the sites of successful commissural splitting. ${ }^{7}{ }^{17} 18$ Surgical data from patients undergoing early mitral valve replacement for severe MR after BMV have shown that the mechanism of severe MR usually involves a leaflet tear associated with commissural calcification. ${ }^{14}{ }^{18}$ Hernandez et al $^{18}$ noted that, as well as commissural calcification, severe subvalvar disease was often found at surgery in patients with severe MR after BMV. Padial et al ${ }^{19}$ showed that a combined echocardiographic assessment of leaflet thickening, subvalvar disease, and commissural calcification was a powerful independent predictor of severe MR with the Inoue technique. In the present study, development of severe MR was associated with a significantly higher Wilkins score. Commissural morphology alone was not a significant predictor, although half the patients developing severe MR had commissural calcification.

\section{Study limitations}

Mean MVA increased from 1.1 to $1.8 \mathrm{~cm}^{2}$, which compares favourably with other studies. However, our success rate after BMV was only $56 \%$. This may be due to the strict criteria we used to define a good outcome: final valve area, percentage gain in valve area, and assessment of MR. MR was assessed by Doppler echocardiography, which is more sensitive than cineventriculography. Our patient group was relatively elderly and more commonly had degenerative valve disease, including commissural calcification. In such patients, symptoms may be usefully palliated, although the gain in MVA is not optimal.

TOE was not repeated routinely after BMV, since it is normally indicated only for the assessment of complications. Therefore, commissural splitting was not directly assessed by TOE. However, BMV is principally a commissurotomy and the association between commissural splitting and successful increase in valve area has been well documented. Although we did not follow up patients in the longer term, previous studies have shown long term outcome to be strongly related to initial improvement in valve area.

\section{Conclusions}

This is the first study to show the value of TOE in assessing mitral commissural fusion and calcification in patients undergoing BMV. These factors form the basis of a novel commissure score, which is reproducible and a useful predictor of immediate outcome after BMV. Application of this scoring system during routine TOE examination before BMV may improve patient selection. Patients with unfavourable commissural morphology (indicated by a commissure 
score of 0 or 1) are less likely to obtain a good result and mitral valve replacement would need to be considered. In cases where surgical risk is prohibitive, we recommend cautious inflations with smaller balloons when commissural calcification has been identified, accepting a suboptimal reduction in gradient.

\section{Authors' affiliations}

N Sutaria, Royal Brompton Hospital, London, UK

T R D Shaw, D Northridge, Western General Hospital, Edinburgh, UK

B Prendergast, Wythenshawe Hospital, Manchester, UK

\section{REFERENCES}

1 Inoue K, Owaki T, Nakamura T, et al. Clinical application of transvenous mitral commissurotomy by a new balloon catheter. J Thorac Cardiovasc Surg 1984:87:394-402.

2 Farhat MB, Ayari M, Maatouk F, et al. Percutaneous balloon versus surgical closed and open mitral commissurotomy: short and long term results. Circulation 1998;97:245-50.

3 Vahanian A, Michel PL, Cormier B, et al. Results of percutaneous mitral commissurotomy in 200 patients. Am J Cardiol 1989;63:847-52.

4 Bassand J, Schiele F, Bernard Y, et al. Double balloon and Inoue techniques in percutaneous mitral valvotomy: comparative results in a series of 232 cases. J Am Coll Cardiol 1991; 18:982-9.

5 Reid CL, McKay CR, Chandraratna PAN, et al. Mechanisms of increase in mitral valve area and the influence of anatomic features in double-balloon catheter balloon valvotomy in adults with rheumatic mitral stenosis: a Doppler and two dimensional echocardiographic study. Circulation 1987;76:628-36.

6 Kaplan JD, Isner JM, Karas RH, et al. In vitro analysis of balloon valvuloplasty of stenotic mitral valves. Am J Cardiol 1987;59:318-23.

7 Fatkin D, Roy P, Morgan J, et al. Percutaneous balloon mitral valvotomy with the Inove single balloon catheter: commissural morphology as a determinant of outcome. J Am Coll Cardiol 1993;21:390-7.
8 Wilkins GT, Weyman AE, Abascal VM, et al. Percutaneous dilatation of the mitral valve: an analysis of echocardiographic variables related to outcome and the mechanism of dilatation. Br Heart J 1988;60:299-308.

9 Abascal VM, Wilkins GT, Choong CY, et al. Echocardiographic evaluation of mitral valve structure and function in patients followed for at least six months after percutaneous mitral balloon valvuloplasty. J Am Coll Cardiol 1988; 12:606-15.

10 Cannan C, Nishimura R, Reeder GS, et al. Echocardiographic assessment of commissural calcium: a simple predictor of outcome after percutaneous mitral balloon valvotomy. J Am Coll Cardiol 1997;29:175-80.

11 Sutaria N, Northridge D, Shaw TRD. The significance of commissural calcification on outcome of mitral balloon valvotomy. Heart 2000;84:398-402.

12 Post JR, Feldman T, Isner J, et al. Inove balloon mitral valvotomy in patients with severe valvular and subvalvular deformity. J Am Coll Cardiol 1995;25:1129-36.

13 Tuzcu EM, Block PC, Griffin B, et al. Immediate and long term outcome of percutaneous mitral valvotomy in patients 65 years and older. Circulation 1992;85:963-71.

14 Cormier B, Vahanian A, Michel PL, et al. Transoesophageal echocardiography in the assessment of percutaneous mitral commissurotomy. Eur Heart J 1991;12(suppl B):61-5.

15 Kronzon I, Tunick OA, Glassman E, et al. Transoesophageal echocardiography to detect atrial clots in candidates for percutaneous transseptal mitral balloon valvuloplasty. J Am Coll Cardiol 1990;16:1320-2.

16 Thomas MR, Monaghan MJ, Smyth DW, et al. Comparative value of transthoracic and transoesophageal echocardiography before balloon dilatation of the mitral valve. Br Heart J 1992;68:493-7.

17 Rittoo D, Sutherland GR, Currie P, et al. The comparative value of transoesophageal and transthoracic echocardiography before and after percutaneous mitral balloon valvotomy: a prospective study. Am Heart J 1993; 125:1094-105

18 Hernandez R, Macaya C, Banuelos C, et al. Predictors, mechanisms and outcome of severe mitral regurgitation complicating mitral valvotomy with the Inove balloon. J Am Coll Cardiol 1992;70:1169-74.

19 Padial LR, Abascal VM, Moreno PR, et al. Echocardiography can predict the development of severe mitral regurgitation after percutaneous mitral valvuloplasty by the Inoue technique. Am J Cardiol 1999;83:1210-3.

\section{IMAGES IN CARDIOLOGY}

\section{Dizziness and dyspnoea: psychiatry and cardiology}

A 58 year old woman was treated for chronic depression with many non-specific thoracic complaints during many years. The previous few months she had complained of progressive dyspnoea. Furthermore she sometimes had "panic attacks" with dyspnoea and dizziness.

Since her chest $x$ ray revealed a moderately enlarged heart silhouette (panel A) she was referred to the cardiologist. An ultrasound examination demonstrated a giant intracardiac tumour in the right atrium (panel B, video 1). Additional coronary angiography demonstrated the vascularisation of the tumour (panel C, video 2) after which it was surgically removed (to view video footage visit the Heart website-http://www.heartjnl.com/supplemental). Pathology confirmed the diagnosis of myxoma. The patient's recovery was uneventful and after six months she experiences less dyspnoea and the dyspnoea attacks and the dizzy spells do not occur as frequently as before.

In psychiatric patients it is often difficult to discriminate cardiac symptoms

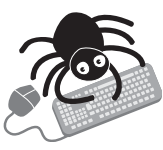

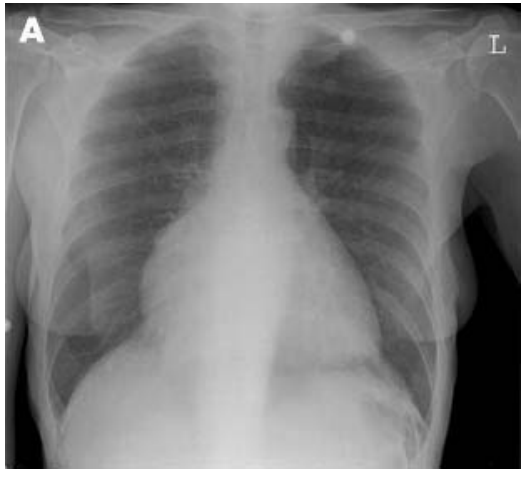

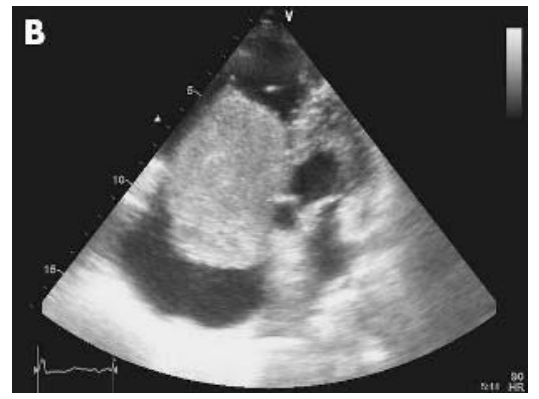

that may be well explained by psychiatric disease. Fortunately in this case a simple chest $x$ ray led to the correct diagnosis.

J P S Henriques, B J Bouma, J J Kloek i.p.henriques@amc.uva.nl To view video footage visit
the Heart website-http:// www.heartinl.com/ supplemental

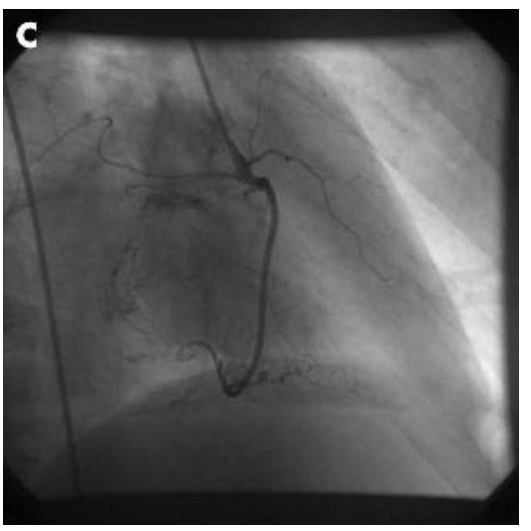

